\title{
ON THE SYMMETRY OF BLOWUP SOLUTIONS TO A MEAN FIELD EQUATION
}

\author{
Chuin Chuan CHEN ${ }^{\text {a }}$, Chang-Shou LIN ${ }^{\text {b }}$ \\ ${ }^{a}$ Department of Mathematics, National Taiwan University, Taipei 106, Taiwan \\ ${ }^{\mathrm{b}}$ Department of Mathematics, Chung-Cheng University, Minghsiung, Chia-Yi 621, Taiwan
}

Received 15 October 1999; revised 15 May 2000

ABSTRACT. - In this article, we consider the mean field equation

$$
\Delta u+\rho\left(\frac{\mathrm{e}^{u}}{\int \mathrm{e}^{u}}-\frac{1}{A}\right)=0 \quad \text { in } \Sigma,
$$

where $\Sigma$ is a flat torus and $A$ is the area of $\Sigma$. This paper is concerned with the symmetry induced by the phenomenon of concentration. By using the method of moving planes, we prove that blowup solutions often possess certain symmetry. In this paper, we consider cases when solutions blowup at one or two points. We also consider related problems for annulu domains of $\mathbf{R}^{2}$.

(C) 2001 L'Association Publications de l'Institut Henri Poincaré. Published by Elsevier B.V. All rights reserved

RÉSUMÉ. - Nous considérons l'équation de champ moyen

$$
\Delta u+\rho\left(\frac{\mathrm{e}^{u}}{\int \mathrm{e}^{u}}-\frac{1}{A}\right)=0 \text { dans } \Sigma,
$$

où $\Sigma$ est un tore plat et $A$ est l'aire de $\Sigma$. Cet article se rapporte à la symétrie induite par le phénomène de concentration. En utilisant la méthode de déplacement de plans, nous démontrons que les solutions avec singularités possèdent dans la plupart des cas des propriétés de symétrie. Dans cet article, nous considérons des cas où les solutions explosent en un ou deux points. Nous traitons également de problèmes reliés pour des domaines annulaires de $\mathbf{R}^{2}$ avec conditions de Dirichlet an berd.

(C) 2001 L'Association Publications de l'Institut Henri Poincaré. Published by Elsevier B.V. All rights reserved

E-mail addresses: chchchen@math.ntu.edu.tw (C.C. Chen), cslin@math.ccu.edu.tw (C.-S. Lin). 


\section{Introduction}

Let $\Sigma$ be a flat torus with a rectangle $\left\{\left(x_{1}, x_{2}\right)|| x_{1}|\leqslant a,| x_{2} \mid \leqslant b\right\}$ of $\mathbf{R}^{2}$ as its fundamental domain. For $\rho>0$, we consider the equation

$$
\Delta u+\rho\left(\frac{\mathrm{e}^{u}}{\int \mathrm{e}^{u}}-\frac{1}{A}\right)=0 \quad \text { in } \Sigma,
$$

where $\Delta$ is the Laplace operator and $A$ is the area of $\Sigma$. Eq. (1.1) is the Euler-Lagrange equation of the following functional

$$
J_{\rho}(u)=\frac{1}{2} \int_{\Sigma}|\nabla u|^{2}+\frac{\rho}{A} \int_{\Sigma} u-\rho \log \int_{\Sigma} \mathrm{e}^{u} .
$$

Clearly Eq. (1.1) and the functional $J_{\rho}$ remain invariant under the adding a constant to $u$. Therefore we always seek solutions of (1.1) which are normalized by

$$
\int_{\Sigma} u \mathrm{~d} x=0 .
$$

Eq. (1.1) is generally known as a mean field equation because it is often derived from various Onsager's vortex theories. Meanwhile, there are many recent works to relate (1.1) to some Chern-Simons-Higgs model. For more information concerning these developments, we refer the interested readers to [4-7,11,12,14,16,20-22,24] and references therein.

For $\rho<8 \pi$, the function $J_{\rho}$ is coercive by the Moser-Trudinger inequality, and solutions of (1.1) can be obtained by minimization of $J_{\rho}$. In fact, even the compactness of solutions can be proved in this case. But, for $\rho \geqslant 8 \pi$, it is quite a different story. Recently, there are several works to extend the existence of (1.1) for $\rho \in(8 \pi, 16 \pi)$. See $[11,12,20,22]$. In [14], Yan-Yan Li initiated a program to find solutions for $\rho \geqslant 8 \pi$ by using the topological degree theory. He proved an uniform bound for solutions to Eq. (1.1) whenever $\rho$ is contained in a compact set of $(8 m \pi, 8(m+1) \pi)$ where $m \geqslant 0$ is an integer. Therefore, the Leray-Schauder degree for (1.1) remains the same when $\rho$ is in the interval $(8 m \pi, 8(m+1) \pi)$. In particular, if the degree is nonvanishing, then the existence of solutions can be guranteed by the degree theory. However, the calculation of the topological degree seems not so easy. One of major difficulties is to prove whether $\rho_{i}>8 m \pi$ or $\rho_{i}<8 m \pi$ for a sequence of solutions of (1.1) with $\lim _{i \rightarrow+\infty} \rho_{i}=8 m \pi$ when blowup would actually happen. In [16], the second author has settled the question when $\Sigma$ is the sphere $S^{2}$, at least for the case of $8 \pi$ and $16 \pi$. The major step in [16] is to show the symmetry induced from the concentration phenomenon. In this paper, we want to continue to study the symmetry of solutions due to the concentration phenomenon. We hope that this would be helpful when we come to computing the topological degree.

In the following, we always suppose that $u_{i}$ is a solution of (1.1) with $\rho=\rho_{i}$ and $u_{i}$ blows up at some points of $\Sigma$. Recall that by a result of [14], if $\rho_{i} \rightarrow 8 m \pi$ and $u_{i}$ blows up somewhere, then $u_{i}$ has exactly $m$ blowup points. 
THEOREM 1.1. - Let $\rho_{i} \rightarrow 8 \pi$ as $i \rightarrow+\infty$ and the center $(0,0)$ of $\Sigma$ is the maximum point of $u_{i}$. Then $u_{i}$ is symmetric with respect to both $x$ and $y$ axis for $i$ large. Furthermore, for any $y$ fixed, $u_{i}(x, y)$ increases when $x$ increases from the negative $x$ up to 0 and decreases when $x$ increases from 0 to the right hand side of the boundary. The same holds for $x$ fixed.

THEOREM 1.2. - Let $\rho_{i} \rightarrow 16 \pi$ as $i \rightarrow+\infty$ and one of local maximum points of $u_{i}$ be located at the center. Then for $i$ large, the other maximum point of $u_{i}$ is located at the corner of the fundamental cell of $\Sigma$. Furthermore, $u_{i}(x, y)$ is symmetric with respect to both $x$ and $y$ axis.

Naturally, we arise the question whether $\rho_{i}$ is larger than $8 \pi$ (or $16 \pi$ ) for solutions with one blowup point (or two blowup points). This question is important when we come to compute the Leray-Schauder degree for $\rho \in(8 \pi, 16 \pi)$. For $S^{2}$, this question has been answered in [16]. For a compact Riemann surface $\Sigma$ of positive genus, the problem remains unsolved. Nevertheless, we have the answer for a similar problem considered in an annulus of $\mathbf{R}^{2}$. Let $\Omega$ be a smooth bounded domain of $\mathbf{R}^{2}$. We consider the following equation.

$$
\begin{cases}\Delta u+\frac{\rho \mathrm{e}^{u}}{\int_{\Omega} \mathrm{e}^{u} \mathrm{~d} x}=0 & \text { in } \Omega, \\ u=0 & \text { on } \partial \Omega .\end{cases}
$$

Eq. (1.4) is derived from Onsager's vortex model for turbulent Euler flows. See [5,6] and [7] for details. For a non-simply-connected domain $\Omega$, the existence of solutions was proved in [12] for $8 \pi<\rho<16 \pi$. In fact, a minimax value $\alpha_{\rho}$ was defined and was proved to be a critical value. In [12], the authors raised the question whether solutions $u_{\rho}$ remains bounded or not as $\rho \rightarrow 16 \pi$, which equivalently is to ask whether solutions of (1.4) is uniformly bounded or not when $\rho$ tends to $16 \pi$ from below. In the following theorem, we answer the question for an annulus domain. For the rest of the section, $\Omega$ is always the annulus $\{x|a<| x \mid<b\}$ for some $a<b$.

THEOREM 1.3. - Let $u_{i}$ be a solution of (1.4) with $\rho_{i} \rightarrow 8 \pi$ as $i \rightarrow+\infty$. Assume that $u_{i}$ has one blowup point, then $\rho_{i}>8 \pi$ for large $i$ and $u_{i}(x)$ is symmetric with respect to the line $l_{i}$, where $l_{i}$ is the line connecting the maximum point of $u_{i}$ and the origin.

For solutions with two blowup points, we have

THEOREM 1.4. - Let $u_{i}$ be a solution of (1.4) with $\rho_{i} \rightarrow 16 \pi$ such that $u_{i}$ blows up at $P$ and $Q$. Assume that $P_{i}$ and $Q_{i}$ are the two local maximum points near $P$ and $Q$ respectively. Then $P_{i}, Q_{i}$ and the origin form a straight line $l_{i}$ and $u_{i}$ is symmetric with respect to $l_{i}$. Moreover, $\rho_{i}>16 \pi$.

COROLLARY 1.5. - Let $\Omega$ be an annulus in $\mathbf{R}^{2}$. For any $\varepsilon>0$, there exists a constant $C=C(\varepsilon)>0$ such that

$$
|u(x)| \leqslant C \quad \text { for } x \in \bar{\Omega}
$$

holds for any solution $u$ of (1.4) with $\rho \in(0,8 \pi] \cup[8 \pi+\varepsilon, 16 \pi]$. 
The paper is organized as follows. In Section 2, both Theorem 1.1 and Theorem 1.2 are proved. Here we apply the well-known method of moving planes to prove our results. For Eq. (1.4) with an annulus $\Omega$, we use the method of rotating planes to prove the axial symmetry of solutions. This is an important step for us to be able to use an isoperimetric inequality of $\mathrm{C}$. Bandle. Together with the isoperimetric inequality of C. Bandle, Theorem 1.3 and Theorem 1.4 are established in Section 3 by this method. For Theorem 1.4, additional results concerning the regular part of Green's function are required. See Lemma 3.3 and Lemma 3.4. We present their proofs here because the authors can not find them in the literature. In the final section, we will construct solutions with $m$ blowup points in the annulus domain for $\rho$ greater then $8 m \pi$.

\section{The method of moving planes}

In this section, we begin with a proof of Theorem 1.1. We use the method of moving planes. For applications of this method, we refer the readers to $[3,10,13,14]$ and [16].

Proof of Theorem 1.1. - After adding a constant, we may assume $u_{i}$ is a solution of

$$
\Delta u_{i}+\rho_{i}\left(\mathrm{e}^{u_{i}}-\frac{1}{A}\right)=0 \quad \text { on } \Sigma .
$$

By translation, we may suppose that the position of the local maximum $P_{i}$ of $u_{i}$ is located at $(a / 2,0)$. For $\lambda>0$, we let $\Sigma_{\lambda}=\left\{x \mid \lambda<x_{1}<\lambda+a\right\}, T_{\lambda}=\left\{x \mid x_{1}=\lambda\right\}$, and $x^{\lambda}=\left(2 \lambda-x_{1}, x_{2}\right)$ is the reflection point of $x$ with respect to $T_{\lambda}$. Set

$$
w_{\lambda}(x)=u_{i}(x)-u_{i}\left(x^{\lambda}\right) \quad \text { for } x \in \Sigma_{\lambda} .
$$

Then $w_{\lambda}(x)$ satisfies

$$
\begin{cases}\Delta w_{\lambda}(x)+b_{\lambda}(x) w_{\lambda}(x)=0 & \text { in } \Sigma_{\lambda} \\ w_{\lambda}(x)=0 & \text { on } \partial \Sigma_{\lambda}\end{cases}
$$

where

$$
b_{\lambda}(x)=\rho_{i} \frac{\mathrm{e}^{u_{i}(x)}-\mathrm{e}^{u_{i}\left(x^{\lambda}\right)}}{u_{i}(x)-u_{i}\left(x^{\lambda}\right)} .
$$

To start the method of moving planes, we need an estimate by Y.Y. Li [14]. Let $\left\{P_{0}^{1}, \ldots, P_{0}^{m}\right\}$ be the blowup set of $u_{i}$. For any $P_{0}^{l}$, we set $P_{i}^{l}$ to be the local maximum point of $u_{i}$ near $P_{0}^{l}$, that is,

$$
u_{i}\left(P_{i}^{l}\right)=\max _{\left|x-P_{0}^{l}\right| \leqslant \delta_{0}} u_{i}(x)
$$

for some small $\delta_{0}>0$. Then the main theorem of [14] is stated as follows.

THEOREM 2.1. - There is a constant $C>0$ such that

$$
\left|u_{i}(x)-U_{i}(x)\right| \leqslant C
$$


holds for $\left|x-P_{i}\right| \leqslant \delta_{0}$ and $i$ large, where $\delta_{0}>0$ is any fixed small number and $U_{i}(x)$ is defined by

$$
U_{i}(x)=\log \frac{\mathrm{e}^{u_{i}\left(P_{i}\right)}}{\left(1+\frac{\rho_{i}}{8} \mathrm{e}^{u_{i}\left(P_{i}\right)}\left|x-P_{i}\right|^{2}\right)^{2}}
$$

for $\left|x-P_{i}\right| \leqslant \delta_{0}$. Furthermore, let $\bar{u}_{i}=-\int_{\Sigma} u_{i}(x) \mathrm{d} x$. Then

$$
u_{i}(x)-\bar{u}_{i} \rightarrow 8 \pi \sum_{l=1}^{m} G\left(\cdot, P_{0}^{l}\right)-\frac{8 \pi m}{A} \int_{\Sigma} G(\cdot, y) \mathrm{d} \mu(y),
$$

in $C^{2}\left(\Sigma \backslash\left\{P_{0}^{1}, \ldots, P_{0}^{m}\right\}\right)$, where $G\left(\cdot, P_{0}^{l}\right)$ is the Green function with a singularity at $P_{0}^{l}$.

From (2.3) and (2.4), we see that

$$
\left|u_{i}(x)+u_{i}\left(P_{i}^{l}\right)\right| \leqslant C
$$

holds when $\left|x-P_{i}^{l}\right| \geqslant \delta_{0}$ for each $l$, and

$$
\left|u_{i}\left(P_{i}^{l^{\prime}}\right)-u_{i}\left(P_{i}^{l}\right)\right| \leqslant C
$$

for $1 \leqslant l^{\prime}, l \leqslant m$. Inequality (2.5) is important for the case of two blowup points in this article.

Now we go back to the proof of Theorem 1.1. We claim

Step 1 .

$$
w_{0}(x)>0 \quad \text { for } x \in \Sigma_{0} .
$$

To prove (2.6), we note that by Theorem 2.1, $\mathrm{e}^{u_{i}(x)} \rightarrow 0$ as $i \rightarrow+\infty$ for $x \in \Sigma \backslash \Sigma_{0}$. Suppose (2.6) is false, that is, the set $\Omega=\left\{x \in \Sigma_{0} \mid w_{0}(x)<0\right\}$ is a non-empty set. Then by $(2.2)$

$$
\Delta w_{0}(x)+\rho_{i} \mathrm{e}^{u_{i}\left(x^{-}\right)} w_{0}(x) \leqslant 0
$$

for $x \in \Omega$ where $x^{-}=\left(-x_{1}, x_{2}\right)$. Therefore the first eigenvalue $\lambda_{1}(\Omega)$ of the linear equation $\Delta+\rho_{i} \mathrm{e}^{u_{i}\left(x^{-}\right)}$on $\Omega$ is nonpositive. On the other hand, since $\mathrm{e}^{u_{i}\left(x^{-}\right)} \rightarrow 0$ as $i \rightarrow+\infty$ and the first eigenvalue of $\Delta$ on $\Sigma_{0}$ is $\pi^{2} / a^{2}$, the first eigenvalue of $\Delta+\rho_{i} \mathrm{e}^{u_{i}\left(x^{-}\right)}$tends to $\pi^{2} / a^{2}$ as $i \rightarrow+\infty$, which yields a contradiction. This proves (2.6).

Set

$$
\lambda_{0}=\sup \left\{\lambda \in[0, a / 2] \mid w_{\tilde{\lambda}}(x) \geqslant 0 \text { for } x \in \Sigma_{\tilde{\lambda}} \text { and } 0 \leqslant \tilde{\lambda} \leqslant \lambda\right\} .
$$

Claim

Step 2. $\lambda_{0}=a / 2$ and $w_{a / 2}(x) \equiv 0$ for $x \in \Sigma_{a / 2}$.

Suppose $\lambda_{0}<a / 2$. Then by the continuity, $w_{\lambda_{0}}(x) \geqslant 0$ for $x \in \Sigma_{\lambda_{0}}$. By the strong maximum principle and the Hopf boundary Lemma, we have

$$
w_{\lambda_{0}}(x)>0 \quad \text { and } \quad \frac{\partial w_{\lambda_{0}}(x)}{\partial v}<0 \quad \text { for } x \in \partial \Sigma_{\lambda_{0}}
$$


where $v$ is the outnormal. By the definition of $\lambda_{0}$, there are a sequence of $\lambda_{j}>\lambda_{0}$ such that $\lim _{j \rightarrow+\infty} \lambda_{j}=\lambda_{0}$ and

$$
w_{\lambda_{j}}\left(x_{j}\right)=\inf _{\Sigma_{\lambda_{j}}} w_{\lambda_{j}}(x)<0,
$$

for some $x_{j} \in \Sigma_{\lambda_{j}}$. After passing to a subsequence, $x_{0}=\lim _{j \rightarrow+\infty} x_{j}$. Then either $x_{0} \in \Sigma_{\lambda_{0}}$ and $w_{\lambda_{0}}\left(x_{0}\right)=0$ or $x_{0} \in \partial \Sigma_{\lambda_{0}}$ and $\nabla w_{\lambda_{0}}\left(x_{0}\right)=0$. Obviously, either case yields a contradiction to (2.7). Hence $\lambda_{0}=a / 2$, and $w_{a / 2}(x) \geqslant 0$ in $\Sigma_{a / 2}$. Of course, we can apply the same procedure from $\lambda=a$ to $\lambda=a / 2$ and obtain $u\left(x^{\lambda}\right) \geqslant u(x)$ also for $x \in \Sigma_{a / 2}$. Thus, we have $u\left(x^{\lambda}\right)=u(x)$ for $\lambda=a / 2$. Clearly, the conclusion of Theorem 1.1 follows immediately.

Now we come to the case of two blowup points. Let $P_{i}$ and $Q_{i}$ are two local maximum points of $u_{i}$ such that both $u_{i}\left(P_{i}\right)$ and $u_{i}\left(Q_{i}\right) \rightarrow+\infty$ as $i \rightarrow+\infty$. Let $P=\lim _{i \rightarrow+\infty} P_{i}$ and $Q=\lim _{i \rightarrow+\infty} Q_{i}$. If there exists an open half $\Sigma^{\prime}$ of the fundamental domain of $\Sigma$ such that $\Sigma^{\prime}$ contains both $P$ and $Q$, then we can apply the method above to yield a contradiction. The reason is as follows. Without loss of generality, we may suppose that $P$ and $Q$ are contained in $\Sigma_{0}$. First, we assume that $P_{i}$ and $Q_{i}$ are not contained in the same vertical line. By step 1 of the proof above, we still have $w_{0}(x)>0$ in $\Sigma_{0}$. And as before, we can move the vertical line until it contains one of the two local maximum points, say $P_{i}$. Since $Q_{i}$ is not on the same vertical line, solutions can not be symmetric with respect to this vertical line. Thus, we can continue our procedure by moving the line to cross $P_{i}$ and $u_{i}$ in the right-hand half is greater than the left-hand half. Clearly, it yields a contradiction to the fact that $P_{i}$ is a local maximum point. If $P_{i}$ and $Q_{i}$ are contained on the same vertical line, then we can move horizontal lines and obtain the same conclusion. Therefore, we conclude that if $P$ is at the center of the fundamental domain, then $Q$ must be at the corner. In the following, we want to prove that for large $i$, if $P_{i}$ is at the center, then $Q_{i}$ must be at the corner.

Proof of Theorem 1.2. - Suppose that $P_{i}$ and $Q_{i}$ are contained in an open half of the fundamental domain of $\Sigma$. Without loss of generality, one may assume that $P_{i}$ and $Q_{i}$ are contained in $(0, a) \times(0, b)$ such that $\lim _{i \rightarrow+\infty} P_{i}=(0,0)$ and $\lim _{i \rightarrow+\infty} Q_{i}=(a, b)$. By translation, we may assume $P_{i}=\left(t_{i}, 0\right), Q_{i}=\left(a_{i}, b_{i}\right)$ with $0<a_{i}<a, 0<b_{i}<b$ and $\left|P_{i}\right|=\left|Q_{i}-(a, b)\right|$. Note that under the assumption of the position of $Q_{i}, u_{i}(x)$ could not be symmetric with respect to $x_{1}$ and $x_{2}$. Let $\delta_{0}$ be a small positive number and $u_{i}\left(P_{i}\right)=\max _{\left|x-P_{i}\right| \leqslant \delta_{0}} u_{i}(x)$ and $u_{i}\left(Q_{i}\right)=\max _{\left|x-Q_{i}\right| \leqslant \delta_{0}} u_{i}(x)$. Then $\left|u_{i}\left(P_{i}\right)-u_{i}\left(Q_{i}\right)\right| \leqslant$ $c$ for some constant $c$ independent of $i$ by (2.5). We want to prove

$$
w_{0}(x)=u_{i}\left(x_{1}, x_{2}\right)-u_{i}\left(-x_{1}, x_{2}\right)>0 \text { for } 0<x_{1}<a .
$$

Once (2.8) is established, we could follow step 2 of Theorem 1.1 to show that $w_{\lambda}(x) \geqslant 0$ for $x \in \Sigma_{\lambda}=\left\{x \mid \lambda<x_{1}<\lambda+a\right\}$ when $0<\lambda \leqslant 2 t_{i}$. Thus, it yields a contradiction to the fact that $u_{i}$ has a local maximum at $P_{i}$. We prove (2.8) by two cases.

Case 1. $\mathrm{e}^{u_{i}\left(P_{i}\right)}\left|t_{i}\right|^{2} \rightarrow+\infty$ as $i \rightarrow+\infty$. 
Note that by (2.5), we also have

$$
\mathrm{e}^{u_{i}\left(Q_{i}\right)}\left|Q_{i}-(a, b)\right|^{2} \rightarrow+\infty \quad \text { as } i \rightarrow+\infty .
$$

For any $x \in \Sigma_{0}$, we have

$$
\mathrm{e}^{u_{i}\left(P_{i}\right)}\left|P_{i}\right|^{2} \leqslant \mathrm{e}^{u_{i}\left(P_{i}\right)}\left|x^{-}-P_{i}\right|^{2} .
$$

Thus, by Theorem 2.1, we have for $x \in B_{\delta_{0}} \cap\left\{x \mid x_{1}>0\right\}$,

$$
\begin{aligned}
u_{i}\left(x^{-}\right) & \leqslant c+\log \frac{\mathrm{e}^{u_{i}\left(P_{i}\right)}}{\left(1+\frac{\rho_{i}}{8} \mathrm{e}^{u_{i}\left(P_{i}\right)}\left|x^{-}-P_{i}\right|^{2}\right)^{2}} \\
& \leqslant c_{1}+\log \left(\frac{1}{\mathrm{e}^{u_{i}\left(P_{i}\right)}\left|x^{-}-P_{i}\right|^{4}}\right),
\end{aligned}
$$

and by (2.9),

$$
\mathrm{e}^{u_{i}\left(x^{-}\right)} \leqslant \mathrm{e}^{c_{1}}\left(\mathrm{e}^{-u_{i}\left(P_{i}\right)}\left|x^{-}-P_{i}\right|^{-4}\right)=\mathrm{o}(1)\left|x^{-}-P_{i}\right|^{-2} \leqslant \mathrm{o}(1)|x|^{-2} .
$$

If $|x| \geqslant \delta_{0}$, then (2.10) holds obviously. Similar inequality holds near $Q_{i}$ also.

Suppose that $w_{0}(x)<0$ for some $x \in \Sigma_{0}$. Without loss of generality, we may assume that the maximum of $-w_{0}$ occurs at some point $x^{0} \in \Sigma_{0}$ with $x_{1}^{0} \leqslant a / 2$. Let $h(x)=\left(x_{1}\right)^{\alpha}$ for $0<\alpha<1$. Clearly, $h(x)$ satisfies

$$
\Delta h(x)=\alpha(\alpha-1)\left(x_{1}\right)^{-2} h(x) .
$$

Set $\bar{w}(x)=-w_{0}(x) / h(x)$. Since $w_{0}$ is $C^{1}$ on $x_{1}=0$, we have $\bar{w}(x)=0$ on $x_{1}=0$. Then by a straightforward computation, $\bar{w}(x)$ satisfies

$$
\left\{\begin{array}{l}
\Delta \bar{w}(x)+2 \nabla(\log h) \cdot \nabla \bar{w}+\left(b_{0}(x)+\alpha(\alpha-1)\left|x_{1}\right|^{-2}\right) \bar{w}(x) \geqslant 0, \\
\bar{w}(x)=0 \quad \text { on } \partial \Omega_{0},
\end{array}\right.
$$

where

$$
b_{0}(x)=\rho_{i} \frac{\mathrm{e}^{u_{i}(x)}-\mathrm{e}^{u_{i}\left(x^{-}\right)}}{u_{i}(x)-u_{i}\left(x^{-}\right)} \quad \text { and } \quad \Omega_{0}=\left\{x \in \Sigma_{0} \mid w_{0}(x)<0\right\} .
$$

Let $y$ be a maximum point of $\bar{w}$. Clearly, $y_{1} \leqslant x_{1}^{0} \leqslant a / 2$, and $b_{0}(y) \leqslant \rho_{i} \mathrm{e}^{u_{i}\left(y^{-}\right)} \leqslant$ $\mathrm{o}(1)|y|^{-2}$ by (2.10). Let $i$ be large enough so that $\mathrm{o}(1)+\alpha(\alpha-1)<0$. Then, applying the maximum principle to (2.12) at $y$, it yields a contradiction. Hence (2.8) is proved in case 1 .

Case 2. Assume $\mathrm{e}^{u_{i}\left(P_{i}\right)}\left|P_{i}\right|^{2} \leqslant c$ for some constant $c>0$.

To prove the positivity of $w_{0}(x)$ in $\Sigma_{0}$, we want to prove

$$
\lim _{i \rightarrow+\infty}\left(\mathrm{e}^{u_{i}\left(P_{i}\right)}|x|^{2}\right)=+\infty \quad \text { and } \quad \lim _{i \rightarrow+\infty} \mathrm{e}^{u_{i}\left(Q_{i}\right)}|x-(a, b)|^{2}=+\infty
$$

for all $x$ whenever $w_{0}(x)<0$. Once (2.13) is proved, we could use $h(x)$ of (2.11) and follow the argument of case 1 to show $w_{0}(x)>0$ for $x \in \Sigma_{0}$. 
Obviously, it suffices to prove (2.13) in a neighborhood of $P_{i}$ and $Q_{i}$. First, suppose $\lim _{i \rightarrow+\infty} \mathrm{e}^{u_{i}\left(P_{i}\right)}\left|P_{i}\right|^{2}>0$. Then we also have $\lim _{i \rightarrow+\infty} \mathrm{e}^{u_{i}\left(Q_{i}\right)}\left|Q_{i}-(a, b)\right|^{2}>0$. In the following, we prove (2.13) for $x$ near $P_{i}$. For the case when $x$ is near $Q_{i}$, the proof is similar. Let

$$
v_{i}(y)=u_{i}\left(\mathrm{e}^{-\frac{u_{i}\left(P_{i}\right)}{2}} y\right)-u_{i}\left(P_{i}\right) .
$$

Then by Theorem 2.1, after passing to a subsequence, $v_{i}(y)$ converges to $v(y) \equiv$ $-2 \log \left(1+\frac{\rho}{8}\left|y-\xi_{0}\right|^{2}\right)$ uniformly in any compact set of $\mathbf{R}^{2}$, where

$$
0 \neq \xi_{0}=\lim _{i \rightarrow+\infty} \mathrm{e}^{\frac{u_{i}\left(P_{i}\right)}{2}} P_{i} \in \mathbf{R}_{+}^{2} .
$$

It is easy to see $w_{0}\left(\mathrm{e}^{-u_{i}\left(P_{i}\right) / 2} y\right)=v_{i}(y)-v_{i}\left(y^{-}\right)$where $y^{-}=\left(-y_{1}, y_{2}\right)$. Thus, by the explicit expression of $v$,

$$
\lim _{i \rightarrow+\infty} w_{0}\left(\mathrm{e}^{-\frac{u_{i}\left(P_{i}\right)}{2}} y\right)=v(y)-v\left(y^{-}\right)>0
$$

for any $y \in \mathbf{R}^{2}$ and $y_{1}>0$. Now suppose there are some $y_{i}$ such that $y_{i}$ is bounded and $w_{0}\left(\mathrm{e}^{-u_{i}\left(P_{i}\right) / 2} y_{i}\right)<0$. Without loss of generality, we may assume $\lim _{i \rightarrow+\infty} y_{i}=$ $y_{0}=\left(y_{0,1}, y_{0,2}\right)$. Since $w_{0}\left(\mathrm{e}^{-u_{i}\left(P_{i}\right) / 2} y\right)$ converges to a positive function for $y_{1}>0$, we have $y_{0,1}=0$. From it, $y_{i}$ can be chosen to be a local minimum point of $w_{0}$ for $\left\{\left(y_{1}, y_{i, 2}\right) \mid 0 \leqslant y_{1} \leqslant 1\right\}$. Hence $\frac{\partial}{\partial y_{1}}\left(v\left(y_{0}\right)-v\left(y_{0}^{-}\right)\right)=0$ at $y=y_{0}$. By a straightforward computation, we have

$$
\frac{\partial}{\partial y_{1}}\left(v\left(y_{0}\right)-v\left(y_{0}^{-}\right)\right)=\frac{-\rho \xi_{0,1}}{1+\frac{\rho}{8}\left|y_{0}-\xi_{0}\right|^{2}}<0
$$

a contradiction. Thus, we have $\left|y_{i}\right| \rightarrow+\infty$ whenever $w_{0}\left(\mathrm{e}^{-u_{i}\left(P_{i}\right) / 2} y_{i}\right) \leqslant 0$. Clearly, (2.13) follows readily. By the similar method, we can prove (2.13) near $Q_{i}$.

Now suppose $\lim _{i \rightarrow+\infty} \mathrm{e}^{u_{i}\left(P_{i}\right)}\left|P_{i}\right|^{2}=0$. Let

$$
N_{i}=\max _{\Sigma_{0}}\left|w_{0}(x)\right|=\left|w_{0}\left(x_{i}\right)\right|
$$

for some $x_{i} \in \Sigma_{0}$. We claim that $x_{i}$ tends to either $(0,0)$ or $(a, \pm b)$ and for any $\delta_{0}>0$,

$$
\sup _{\Sigma_{0}\left(\delta_{0}\right)}\left|w_{0}(x)\right|=\mathrm{o}(1) N_{i}
$$

where $\Sigma_{0}\left(\delta_{0}\right)=\left\{x \in \Sigma_{0}|| x-P_{i} \mid \geqslant \delta_{0}\right.$ and $\left.\left|x-Q_{i}\right| \geqslant \delta_{0}\right\}$.

We prove (2.15) by contradiction. Assume that for a small $\delta_{0}>0$, there are subsequence of $u_{i}$ (still denoted by $u_{i}$ ) such that

$$
\sup _{\Sigma_{0}\left(\delta_{0}\right)}\left|w_{0}(x)\right| \geqslant c_{0} N_{i}
$$

holds for some constant $c_{0}$. Set $\hat{w}_{i}(x)=N_{i}^{-1} w_{0}(x)$. Then $\left|\hat{w}_{i}(x)\right| \leqslant 1$ for $x \in \bar{\Sigma}_{0}$. Since $b_{0}(x) \rightarrow 0$ uniformly in any compact set of $\bar{\Sigma} \cap \bar{\Sigma}_{0} \backslash\{0,(a, \pm b)\}$, by the elliptic 
estimates, there are a subsequence of $\hat{w}_{i}$ (still denoted by $\hat{w}_{i}$ ) such that $\hat{w}_{i}$ converges to a harmonic function $\hat{w}$, where $\hat{w}$ is smooth in $\bar{\Sigma} \cap \bar{\Sigma}_{0}$ except for the points $(0,0)$, $(a, \pm b)$. Since $|\hat{w}(x)| \leqslant 1$ in $\bar{\Sigma}_{0}$ and $\hat{w}(x)=0$ for $x \in\left(\partial \Sigma_{0} \backslash\{(0,0),(a, \pm b)\}\right) \cap \bar{\Sigma}$, by the reflection and the elliptic regularity for bounded harmonic functions, we conclude that $\hat{w}(x)$ is smooth for $x \in \bar{\Sigma}_{0}$ and then, $\hat{w}(x) \equiv 0$ for $x \in \Sigma_{0}$. However, it obviously contradicts to the assumption (2.16). Hence the claim (2.15) is proved.

Now suppose that the maximum of $w_{0}$ occures in a neighborhood of $P_{i}$. If the maximum of $w_{0}$ occures near $Q_{i}$, we can prove (2.13) by the same argument as follows. Define $v_{i}$ as (2.14), and $\tilde{w}_{0}$ by

$$
\tilde{w}_{0}(y)=w_{0}\left(\mathrm{e}^{-\frac{u_{i}\left(P_{i}\right)}{2}} y\right)
$$

for $|y| \leqslant \mathrm{e}^{u_{i}\left(P_{i}\right) / 2} \delta_{0}$ for some fixed number $\delta_{0}>0$. Then $\tilde{w}_{0}$ satisfies

$$
\Delta \tilde{w}_{0}+\tilde{b}(y) \tilde{w}_{0}(y)=0 \quad \text { for }|y| \leqslant \mathrm{e}^{u_{i}\left(P_{i}\right) / 2} \delta_{0},
$$

where

$$
\tilde{b}(y)=\frac{\mathrm{e}^{v_{i}(y)}-\mathrm{e}^{v_{i}\left(y^{-}\right)}}{v_{i}(y)-v_{i}\left(y^{-}\right)} .
$$

By Theorem 2.1, $\tilde{b}(y)$ is bounded by

$$
|\tilde{b}(y)| \leqslant A\left(1+|y|^{2}\right)^{-2}
$$

for $|y| \leqslant \mathrm{e}^{u_{i}\left(P_{i}\right) / 2} \delta_{0}$ and for some constant $A$. Applying the Green representation formula, we have

$$
\left|\tilde{w}_{0}(x)\right| \leqslant \frac{1}{2 \pi} \int_{B_{i}} \log \frac{\left|x^{-}-y\right|}{|x-y|}\left|\tilde{b}_{0}(y)\right|\left|\tilde{w}_{0}(y)\right|+\mathrm{o}(1) N_{i},
$$

where $B_{i}=\left\{y \mid y_{1} \geqslant 0\right.$ and $\left.|y| \leqslant \mathrm{e}^{u_{i}\left(P_{i}\right) / 2} \delta_{0}\right\}$,

$$
G_{0}(x, y)=\frac{1}{2 \pi} \log \frac{\left|x^{-}-y\right|}{|x-y|}
$$

is the Green function for the half plane $\left\{y \mid y_{1}>0\right\}$, and the term o(1) $N_{i}$ comes from the boundary value of $\tilde{w}_{0}$ and (2.15). Let $y_{i}$ be the maximum point of $\tilde{w}_{0}$. Then by using (2.18), we estimate the integral of the right hand side of (2.19) by $\left(1+\left|y_{i}\right|\right)^{-1} N_{i}$. Thus, (2.19) implies

$$
N_{i} \leqslant c\left(1+\left|y_{i}\right|\right)^{-1} N_{i},
$$

which implies $y_{i}$ is bounded. Let $\lim _{i \rightarrow+\infty} y_{i}=y_{0}$. Then by elliptic estimates, there are a subsequence of $N_{i}^{-1} \tilde{w}_{0}$ such that $N_{i}^{-1} \tilde{w}_{0}$ converges to $w \not \equiv 0$ in $C_{\text {loc }}^{2}$ which satisfies

$$
\begin{cases}\Delta w+\rho \mathrm{e}^{v(y)} w=0 & \text { for } y_{1} \geqslant 0 \\ w(y)=0 & \text { for } y_{1}=0 .\end{cases}
$$


By (2.19), we have

$$
\left|\tilde{w}_{0}(y)\right| \leqslant c N_{i}(1+|y|)^{-1}+\mathrm{o}(1) N_{i}
$$

for $|y| \leqslant \mathrm{e}^{u_{i}\left(P_{i}\right) / 2} \delta_{0}$. Hence $|w(y)| \leqslant c(1+|y|)^{-1}$, and then by Lemma 2.2 below, $w(y)=c \frac{\partial v}{\partial y_{1}}(y)$ for some constant $c \neq 0$. Let $\xi_{i}=\mathrm{e}^{u_{i}\left(P_{i}\right) / 2} P_{i}$. Since $\tilde{w}_{0}\left(\xi_{i}\right)>0$ and $\frac{\partial v}{\partial y_{1}}<0$ for $y_{1}>0$, we have $c<0$. Hence, on any compact set, $\tilde{w}_{0}(y)>0$ for $i$ large. This proves (2.13) near $P_{i}$.

To prove (2.13) near $Q_{i}$, we note that

$$
N_{i}^{-1} \frac{\partial \tilde{w}_{0}}{\partial y_{1}}\left(\xi_{i}\right)=N_{i}^{-1} \frac{\partial v_{i}}{\partial y_{1}}\left(\xi_{i}^{-}\right)=N_{i}^{-1} \frac{\partial^{2} v_{i}}{\partial y_{1}^{2}}\left(\eta_{i}\right)\left(-2 \xi_{i, 1}\right),
$$

where $\frac{\partial v_{i}}{\partial y_{1}}\left(\xi_{i}\right)=0$ is used and $\left|\eta_{i}\right| \leqslant\left|\xi_{i}\right|$. Since $\frac{\partial^{2} v}{\partial y_{1}^{2}}(0)<0$, we have

$$
\lim _{i \rightarrow+\infty} N_{i}^{-1} \cdot \xi_{i, 1}=\lim _{i \rightarrow+\infty} N_{i}^{-1} \mathrm{e}^{\frac{u_{i}\left(P_{i}\right)}{2}}\left|P_{i}\right|>0
$$

By (2.21), we have

$$
\lim _{i \rightarrow+\infty} N_{i}^{-1} \mathrm{e}^{\frac{u_{i}\left(Q_{i}\right)}{2}}\left|Q_{i}-(a, b)\right|>0
$$

also. Thus, by using calculation similar to (2.20) near $Q_{i}$, we have

$$
\lim _{i \rightarrow+\infty} N_{i}^{-1}\left|\nabla w_{0}^{*}\left(\xi_{i}^{*}\right)\right|=\lim _{i \rightarrow+\infty} N_{i}^{-1} \mathrm{e}^{\frac{u_{i}\left(Q_{i}\right)}{2}}\left|Q_{i}-(a, b)\right|>0,
$$

where

$$
w_{0}^{*}(y)=w_{0}\left((a, b)+\mathrm{e}^{-\frac{u_{i}\left(Q_{i}\right)}{2}} y\right) \quad \text { and } \quad \xi_{i}^{*}=\mathrm{e}^{\frac{u_{i}\left(Q_{i}\right)}{2}}\left(Q_{i}-(a, b)\right) .
$$

Therefore, $N_{i}^{-1} w_{0}^{*}(y)$ converges to a nonzero limit. Since $Q_{i}$ is a local maximum, the limiting function is positive on $\mathbf{R}_{+}^{2}$. Thus, for any compact set of $\overline{\mathbf{R}}_{+}^{2}, N_{i}^{-1} w_{0}^{*}(y)$ is positive on any compact set of $\mathbf{R}_{+}^{2}$ and for $i$ large. This proves (2.13) near $Q_{i}$.

By (2.13), $w_{0}(x)>0$ for $x \in \Sigma_{0}$. Thus, by moving the line $x_{1}=\mu$ cross $t_{i}$, we prove $w_{\mu}(x)>0$ in $\Sigma_{\mu}$ for $0 \leqslant \mu \leqslant 2 t_{i}$. Clearly, it yields a contradiction. Therefore, the positions of $P_{i}$ and $Q_{i}$ are proved as claimed.

To prove the symmetry with respect to $x$, we consider $P_{i}=(0,0), Q_{i}=(a, b)$ and set $w_{0}(x)=u_{i}\left(x_{1}, x_{2}\right)-u_{i}\left(-x_{1}, x_{2}\right)$ for $x \in \Sigma_{0}$. Suppose $w_{0}(x) \not \equiv 0$ for $x \in \Sigma_{0}$. Then as the proof in case $2,(2.15)$ holds for any $\delta_{0}>0$. Thus, the maximum of $\left|w_{0}(x)\right|$ can occur only near either $P_{i}$ or $Q_{i}$ (not like the situation in case $2, w_{0}(x)$ may not simultaneously be positive near $P_{i}$ and near $\left.Q_{i}\right)$. Suppose the maximum of $\left|w_{0}(x)\right|$ occures near $P_{i}$. Then by the Green formula (2.19), $\tilde{w}_{i}(y)=N_{i}^{-1} w\left(\mathrm{e}^{-u_{i}\left(P_{i}\right) / 2} y\right)$ converges to $w(y)$ in $C_{\mathrm{loc}}^{2}\left(\mathbf{R}_{+}^{2}\right)$, where $w(y)$ satisfies

$$
\begin{cases}\Delta w+\rho \mathrm{e}^{v(y)} w=0 & \text { in } \mathbf{R}_{+}^{2}=\left\{\left(y_{1}, y_{2}\right) \mid y_{1} \geqslant 0\right\} \\ w\left(y_{1}, 0\right)=0, & y_{1} \in \mathbf{R}\end{cases}
$$


and $|w(y)| \leqslant c(1+|y|)^{-1}$. By the reflection and Lemma 2.2, $w(y)=c \frac{\partial v}{\partial y_{1}}$ for some constant $c \neq 0$. But, since $\nabla \tilde{w}_{i}(0)=2 \nabla v_{i}(0)=0$, we have $0=\frac{\partial w}{\partial y_{1}}(0)=c \frac{\partial^{2} v}{\partial y_{1}^{2}}(0)=\frac{\rho c}{2}$, which implies $c=0$, a contradiction. Thus, the maximum $\left|w_{0}(x)\right|$ must occur near $Q_{i}$. But the same argument is applied to yield a contradiction when the maximum point is near $Q_{i}$. Therefore, $w_{0}(x)$ must vanish completely in $\Sigma_{0}$. The symmetry of $u_{i}$ follows readily.

LEMMA 2.2. - Let $\varphi(x)$ be a solution of

$$
\Delta \varphi+\mathrm{e}^{v(x)} \varphi=0 \quad \text { in } \mathbf{R}^{2},
$$

where $v(x)=-2 \log \left(1+|x|^{2} / 8\right)$. Suppose that $\varphi(x)$ is bounded in $\mathbf{R}^{2}$. Then

$$
\varphi(x)=\sum_{j=0}^{2} a_{j} \psi_{j}(x)
$$

for some constants $a_{j} \in \mathbf{R}$, where $\psi_{j}(x)=\left(1+|x|^{2} / 8\right)^{-1} x_{j}$ for $j=1,2$ and $\psi_{0}(x)=$ $\left(1+|x|^{2} / 8\right)^{-1}\left(1-|x|^{2} / 8\right)$.

Proof. - Set $\varphi_{k}(r)=\frac{1}{2 \pi} \int_{0}^{2 \pi} \varphi(x) \cos k \theta \mathrm{d} \theta$ for $k \geqslant 1$. Then $\varphi_{k}$ satisfies

$$
\left\{\begin{array}{l}
\Delta \varphi_{k}(r)-\frac{k^{2}}{r^{2}} \varphi_{k}(r)+\mathrm{e}^{v(r)} \varphi_{k}(r)=0 \\
\varphi_{k}(0)=0
\end{array}\right.
$$

Let $\tilde{\varphi}_{1}=\frac{1}{2 \pi} \int_{0}^{2 \pi} \psi_{1}(x) \cos \theta \mathrm{d} \theta$. Then $\tilde{\varphi}_{1}$ satisfies (2.23) for $k=1$. By the uniquencess of solutions of ODE, $\varphi_{1}(r)=c \tilde{\varphi}_{1}(r)$ for some constant $c \in \mathbf{R}$.

Suppose $\varphi_{k}(r) \not \equiv 0$ for some $k \geqslant 2$. Since $\tilde{\varphi}_{1}(r)>0$, then, by the comparison with $\tilde{\varphi}_{1}(r), \varphi_{k}(r)$ never vanishes for $r>0$ and $k \geqslant 2$. By the assumption, $\varphi_{k}(r)$ is bounded. By an elementary argument and (2.22), $r \varphi_{k}^{\prime}(r) \rightarrow 0$ as $r \rightarrow+\infty$. Comparing with $\tilde{\varphi}_{1}$, we have,

$$
0=\int_{0}^{\infty}\left(\tilde{\varphi}_{1} \Delta \varphi_{k}-\varphi_{k} \Delta \tilde{\varphi}_{1}\right) r \mathrm{~d} r=\left(k^{2}-1\right) \int_{0}^{\infty} \frac{\varphi_{k}(r) \tilde{\varphi}_{1}(r)}{r} \mathrm{~d} r,
$$

a contradiction since $\varphi_{k}(r) \tilde{\varphi}_{1}(r)$ has only one sign. Then, $\varphi_{k}(r) \equiv 0$ for $k \geqslant 2$. The conclusion of Lemma 2.2 follows.

\section{Mean field equation on annulus domains}

In this section, we consider a sequence of solutions $u_{i}$ of

$$
\begin{cases}\Delta u_{i}+\frac{\rho_{i} e_{i}}{\int_{\Omega} \mathrm{e}^{u_{i}} \mathrm{~d} x}=0 & \text { in } \Omega, \\ u_{i}=0 & \text { on } \partial \Omega,\end{cases}
$$

where $\Omega=\{x|a<| x \mid<b\}$ for some $a<b$. Suppose that $u_{i}$ has one single blow up point at $P$ and $\max _{\bar{\Omega}} u_{i}=u_{i}\left(P_{i}\right) \rightarrow+\infty$ as $i \rightarrow+\infty$. Without loss of generality, we 
may assume $P_{i}=\left(r_{i}, \pi / 2\right)$ in the polar coordinate. Following the proof of Theorem 1.1, we can show that

$$
w_{0}(x)=u_{i}(x)-u_{i}\left(x^{-}\right)>0
$$

for $x_{2}>0$, where $x^{-}=\left(x_{1},-x_{2}\right)$. Instead of the method of moving planes which was used in the second step of the proof of Theorem 1.1, we use the method of "rotating planes". For any $\theta \in[0, \pi / 2]$, let $l_{\theta}$ denote the line $\{(t \cos \theta, t \sin \theta) \mid t \in \mathbf{R}\}$ and $\Sigma_{\theta}$ be one of the components of $\Omega \backslash l_{\theta}$ such that $P_{i} \in \Sigma_{\theta}$. Set

$$
w_{\theta}=u(x)-u\left(x^{\theta}\right),
$$

where $x^{\theta}$ is the reflection point of $x$ with respect to $l_{\theta}$. For $\theta=0$, we have $w_{0}(x)>0$ for $x \in \Sigma_{0}$ by (3.1). Set

$$
\theta_{0}=\sup \left\{\theta \in[0, \pi / 2] \mid w_{\tilde{\theta}}(x)>0 \text { for } x \in \Sigma_{\tilde{\theta}} \text { and } 0 \leqslant \tilde{\theta}<\theta\right\} .
$$

By the argument similar to step 2 of the proof of Theorem 1.1, we can prove $\theta_{0}=\pi / 2$ and $w_{\pi / 2}(x) \equiv 0$, that is, $u_{i}(x)$ is symmetric with respect to $x_{1}$, and

$$
w_{\theta}(x)>0 \text { for } x \in \Sigma_{\theta} \text { and } 0 \leqslant \theta<\frac{\pi}{2} .
$$

By the Hopf lemma and (3.3), we have

$$
\frac{\partial w_{\theta}}{\partial v_{\theta}}(x)>0 \text { for } x \in \partial \Sigma_{\theta} \cap l_{\theta},
$$

where $v_{\theta}(x)$ is the outnormal at $x \in \partial \Sigma_{\theta} \cap l_{\theta}$. Obviously, by (3.4), we have

$$
\frac{\partial u_{i}}{\partial \theta}(x)=x_{1} \frac{\partial u_{i}}{\partial x_{2}}(x)-x_{2} \frac{\partial u_{i}}{\partial x_{1}}(x)>0 \quad \text { for } x_{1}>0 .
$$

Note that $\partial u_{i} / \partial \theta$ is odd in $x_{1}$. Hence $\partial u_{i}(x) / \partial \theta=0$ on $x_{1}=0$, which implies we have

LEMMA 3.1. - Let $\phi=\partial u_{i} / \partial \theta$. Then $\phi$ satisfies

$$
\begin{cases}\Delta \phi+\rho_{i} \mathrm{e}^{v_{i}} \phi=0 & \text { in } \Omega^{+}, \\ \phi(x)>0 & \text { in } \Omega_{+} \text {and } \phi=0 \text { on } \partial \Omega^{+},\end{cases}
$$

where $\Omega^{+}=\left\{\left(x_{1}, x_{2}\right) \in \Omega \mid x_{1} \geqslant 0\right\}$ and $v_{i}=u_{i}-\log \left(\int_{\Omega} \mathrm{e}^{u_{i}}\right)$.

To estimate $\rho_{i}$, we need an isoperimetric inequality which was due to C. Bandle. See [1].

LEMMA 3.2. - Suppose $u$ is a solution of $\Delta u+\rho \mathrm{e}^{u}=0$ in $\omega$, where $\omega$ is a simply connected domain. If $\rho \int_{\omega} \mathrm{e}^{u} \mathrm{~d} x<4 \pi$, then the first eigenvalue of $\Delta+\rho \mathrm{e}^{u}$ for the Dirichlet problem is positive.

Clearly, $\rho_{i} \geqslant 8 \pi$ by Lemma 3.1 and Lemma 3.2. However, we require a stronger version of Lemma 3.2 to ensure $\rho_{i}>8 \pi$. In order to see whether Lemma 3.2 holds or 
not when $\rho \int_{\omega} \mathrm{e}^{u} \mathrm{~d} x=4 \pi$, we have to go back to the proof of Lemma 3.2. Since the proof is not very long, we present here for the completeness.

Proof of Theorem 1.3. - Let $v_{i}=u_{i}+c_{i}$ for some constant $c_{i}$ such that $v_{i}$ satisfies $\Delta v_{i}+\mathrm{e}^{v_{i}}=0$ in $\Omega$ and $\phi=\partial v_{i} / \partial \theta$. Then $\rho_{i}=\int_{\Omega} \mathrm{e}^{v_{i}} \mathrm{~d} x \rightarrow 8 \pi$ as $i \rightarrow+\infty$. Set $\Omega^{+}=\left\{\left(x_{1}, x_{2}\right) \mid x_{1}>0\right\}$. So, $\phi(x)>0$ in $\Omega^{+}$, and $\phi(x)=0$ on $\partial \Omega^{+}$. Let $v(x)=$ $v(|x|)=-2 \log \left(1+\frac{1}{8}|x|^{2}\right)$ be the solution of

$$
\left\{\begin{array}{l}
\Delta v+\mathrm{e}^{v}=0 \quad \text { in } \mathbf{R}^{2} \\
\int_{B_{1}} \mathrm{e}^{v} \mathrm{~d} x=4 \pi
\end{array}\right.
$$

Now assume $\int_{\Omega^{+}} \mathrm{e}^{v_{i}} \mathrm{~d} x=4 \pi$. Let $\Omega_{t}^{+}=\left\{x \in \Omega^{+} \mid \phi(x)>t\right\}$ for $t>0$. Set $B_{t}^{*}$ be the open ball with center 0 such that

$$
\int_{B_{t}^{*}} \mathrm{e}^{v} \mathrm{~d} x=\int_{\Omega_{t}^{+}} \mathrm{e}^{v_{i}} \mathrm{~d} x=A(t) .
$$

Define the spherically decreasing rearrangement $\phi^{*}$ of $\phi$ to be a non-negative function in $B_{1}$ such that

$$
\phi^{*}(x)=\phi^{*}(|x|)=\sup \left\{t \mid x \in B_{t}^{*}\right\} .
$$

Obviously, $\phi^{*}(x)>t_{0}$ if and only if $x \in B_{t_{0}}^{*}$ (here we use the fact that $B_{t}^{*}$ is open). Thus, $\phi^{*}(x)$ has the same distribution function (with respect to the measure $\mathrm{e}^{v} \mathrm{~d} x$ ) as $\phi$ (with respect to the measure $\mathrm{e}^{v_{i}} \mathrm{~d} x$ ). So, the identity

$$
\int_{B_{1}} \phi^{* 2} \mathrm{e}^{v} \mathrm{~d} x=\int_{\Omega^{+}} \phi^{2} \mathrm{e}^{v_{i}} \mathrm{~d} x
$$

holds. To prove

$$
\int_{\Omega^{+}}|\nabla \phi|^{2} \mathrm{~d} x \geqslant \int_{B_{1}}\left|\nabla \phi^{*}\right|^{2} \mathrm{~d} x .
$$

We need Bol's inequality which states that for any $\omega \subset \subset \Omega^{+}$,

$$
\frac{1}{2}(8 \pi-A(\omega)) A(\omega) \leqslant l^{2}(\partial \omega),
$$

provided that $A\left(\Omega^{+}\right) \leqslant 8 \pi$, where

$$
A(\omega)=\int_{\omega} \mathrm{e}^{v_{i}} \mathrm{~d} x \quad \text { and } \quad l(\partial \omega)=\int_{\partial \omega} \mathrm{e}^{\frac{1}{2} v_{i}} \mathrm{~d} \sigma .
$$

We note that the equality of (3.12) holds when the measure $\mathrm{e}^{v} \mathrm{~d} x$ is used and $\omega$ is a ball with center 0 .

Since $\phi$ satisfies an elliptic equation, it is easy to prove the Lebesgue measure of $\partial \Omega_{t}$ is equal to zero for any $t>0$. Thus, $A(t)$ is continuous and strictly decreasing it $t$. By 
the co-area formulas,

$$
-A^{\prime}(t)=\int_{\{\phi=t\}} \frac{\mathrm{e}^{v_{i}}}{|\nabla \phi|} \mathrm{d} s \quad \text { a.e. } t>0,
$$

and

$$
-\frac{\mathrm{d}}{\mathrm{d} t}\left(\int_{\Omega_{t}^{+}}|\nabla \phi|^{2} \mathrm{~d} x\right)=\int_{\{\phi=t\}}|\nabla \phi| \mathrm{d} s \quad \text { a.e. } t>0
$$

hold. By the Schwarz inequality and Bol's inequality, we have

$$
\begin{aligned}
-\frac{\mathrm{d}}{\mathrm{d} t}\left(\int_{\Omega_{t}^{+}}|\nabla \phi|^{2} \mathrm{~d} x\right) & \geqslant\left(\int_{\{\phi=t\}} \mathrm{e}^{v_{i} / 2} \mathrm{~d} x\right)^{2}\left(\int_{\{\phi=t\}} \frac{\mathrm{e}^{v_{i}}}{|\nabla \phi|} \mathrm{d} x\right)^{-1} \\
& =l^{2}\left(\partial \Omega_{t}^{+}\right)\left(-A^{\prime}(t)\right)^{-1} \\
& \geqslant \frac{1}{2}(8 \pi-A(t)) A(t)\left(-A^{\prime}(t)\right)^{-1} \\
& =-\frac{\mathrm{d}}{\mathrm{d} t}\left(\int_{B_{t}^{*}}\left|\nabla \phi^{*}\right|^{2} \mathrm{~d} x\right)
\end{aligned}
$$

for a.e. $t>0$, where the equality of (3.12) is used for the metric $\mathrm{e}^{v}|\mathrm{~d} x|^{2}$. Integrating (3.15), one has

$$
\int_{\Omega^{+}}|\nabla \phi|^{2} \mathrm{~d} x \geqslant \int_{B_{1}}\left|\nabla \phi^{*}\right|^{2} \mathrm{~d} x
$$

as claimed by (3.11).

Together (3.10) and (3.11), we have

$$
\int_{B_{1}}\left|\nabla \phi^{*}\right|^{2}-\int_{B_{1}} \phi^{* 2} \mathrm{e}^{v} \mathrm{~d} x \leqslant \int_{\Omega^{+}}|\nabla \phi|^{2}-\int_{\Omega^{+}} \phi^{2} \mathrm{e}^{v_{i}} \mathrm{~d} x=0 .
$$

Since the first eigenvalue of $\Delta+\mathrm{e}^{v}$ for the Dirichlet problem is equal to zero, (3.17) implies

$$
\int_{B_{1}}\left|\nabla \phi^{*}\right|^{2}-\int_{B_{1}} \phi^{* 2} \mathrm{e}^{v} \mathrm{~d} x=0,
$$

that is, each inequality in (3.15) must be an equality. In particular,

$$
\mathrm{e}^{v_{i}}=\Phi_{i}(\phi)|\nabla \phi|^{2}
$$

holds for some function $\Phi_{i}$. Clearly, $\Phi_{i}$ is continuous at $\phi=t$ as long as $t$ is not a critical value of $\phi$. Since $\phi=0$ is not a critical value of $\phi$, one has $|\nabla \phi|(x) \equiv$ constant whenever $|x|=b$ or $|x|=a$. Since $\partial \phi / \partial \theta \equiv 0$ for $x \in \partial \Omega, \phi_{r}(b, \theta)$ is a constant independent of $\theta$. 
However,

$$
\int_{0}^{2 \pi} \phi_{r}(b, \theta) \mathrm{d} \theta=\int_{0}^{2 \pi}\left(u_{r}\right)_{\theta}(b, \theta) \mathrm{d} \theta=0,
$$

which implies $|\nabla \phi(x)| \equiv 0$ on $\partial \Omega$. Clearly, it yields a contradiction to the Hopf boundary lemma.

Let $G(x, y)$ denote the Green function of $\Delta$ with vanishing boundary volue. Set

$$
\begin{gathered}
\psi(x, y)=G(x, y)-\frac{1}{2 \pi} \log \frac{1}{|x-y|}, \quad \text { and } \\
\varphi(x)=\psi(x, x)
\end{gathered}
$$

is the regular part of the Green function. Recall that $\Omega=\{x|a<| x \mid<b\}$.

LEMMA 3.3. - Let $\Omega$ be the radially symmetric annulus $\{x|a<| x \mid<b\}$ and $\varphi(x)$ be the regular part of the Green function. Then $\varphi(x)=\varphi(|x|)$ and $\varphi(r)$ has a unique critical point $r_{0}$. Furthermore, $r_{0}$ is the maximum point and $\varphi^{\prime \prime}\left(r_{0}\right)<0$.

Proof. - By the uniqueness of the Green function, it is easy to see that $\varphi(x)$ is radially symmetric. Since $\lim _{x \rightarrow \partial \Omega} \varphi(x)=-\infty, \varphi(r)$ has a critical point $r_{0}$ at least. We want to prove $\varphi^{\prime \prime}\left(r_{0}\right)<0$ for any critical point $r_{0}$. Then Lemma 3.3 follows readily.

Let $y_{0}=r_{0} e_{2}=\left(0, r_{2}\right)$. Since $\psi(x, y)=\psi(y, x)$, we have

$$
\nabla_{x} \psi\left(y_{0}, y_{0}\right)=\nabla_{y} \psi\left(y_{0}, y_{0}\right)=0
$$

where

$$
\nabla_{x} \psi(x, y)=\left(\frac{\partial \psi}{\partial x_{1}}(x, y), \frac{\partial \psi}{\partial x_{2}}(x, y)\right)
$$

is the first derivative of $\psi$ with respect to $x$-variable. Also, we have

$$
\psi\left(x, y_{0}\right)=\psi\left(x^{-}, y_{0}\right),
$$

where $x^{-}=\left(-x_{1}, x_{2}\right)$. Set

$$
\frac{\partial \psi}{\partial \theta}\left(x, y_{0}\right)=x_{1} \frac{\partial \psi}{\partial x_{2}}\left(x, y_{0}\right)-x_{2} \frac{\partial \psi}{\partial x_{1}}\left(x, y_{0}\right) .
$$

Note that $\psi\left(x, y_{0}\right)=\frac{1}{2 \pi} \log \left|x-y_{0}\right|$ for $x \in \partial \Omega$. Together with (3.22), $\frac{\partial \psi}{\partial \theta}\left(x, y_{0}\right)$ satisfies

$$
\frac{\partial \psi}{\partial \theta}\left(x, y_{0}\right) \leqslant 0 \quad \text { for } x \in \partial \Omega^{+},
$$

where $\Omega^{+}=\left\{x \in \Omega \mid x_{1}>0\right\}$. By the maximum principle, $\frac{\partial \psi}{\partial \theta}\left(x, y_{0}\right)<0$ for $x \in \Omega^{+}$. Thus,

$$
\psi\left(|x| e_{2}, y_{0}\right) \leqslant \psi\left(x, y_{0}\right)
$$


for $x \in \Omega$. By (3.21) and the maximum principle, we have

$$
\frac{\partial^{2} \psi}{\partial x_{1}^{2}}\left(y_{0}, y_{0}\right) \geqslant 0
$$

which implies

$$
\frac{\partial^{2} \psi}{\partial x_{2}^{2}}\left(y_{0}, y_{0}\right) \leqslant 0
$$

On the other hand, $\frac{\partial \psi}{\partial y_{2}}\left(x, y_{0}\right)$ is a harmonic function which by a straightforward computation, satisfies

$$
\frac{\partial \psi}{\partial y_{2}}\left(x, y_{0}\right) \begin{cases}>0 & \text { if }|x|=a \text { or } x=(b \cos \theta, b \sin \theta) \text { with }-\pi / 2 \leqslant \theta<\theta_{0}, \\ <0 & \text { if } x=(b \cos \theta, b \sin \theta) \text { with } \theta_{0}<\theta \leqslant \pi / 2\end{cases}
$$

where $\sin \theta_{0}=r_{0} / b$. Note that $\frac{\partial \psi}{\partial y_{2}}\left(x, y_{0}\right)=\frac{\partial \psi}{\partial y_{2}}\left(x^{-}, y_{0}\right)$. Hence the nodal line $\{x \mid$ $\left.\frac{\partial \psi}{\partial y_{2}}\left(x, y_{0}\right)=0\right\}$ must intersect with the positive $x_{2}$-axis only at $x=r_{0} e_{2}$. Otherwise, by the symmetry of $\frac{\partial \psi}{\partial y_{2}}\left(x, y_{0}\right)$ in $x_{1}$, the nodal line would enclose a region in $\Omega$, which violates the maximum principle. Therefore, by (3.24) and (3.21),

$$
\begin{array}{ll}
\frac{\partial \psi}{\partial y_{2}}\left(r_{0} e_{2}, y_{0}\right)=0 \quad \text { and }, \\
\frac{\partial \psi}{\partial y_{2}}\left(r e_{2}, y_{0}\right)<0 \quad \text { for } r_{0}<r \leqslant b .
\end{array}
$$

By the Hopf lemma, one has

$$
\frac{\partial^{2} \psi}{\partial x_{2} \partial y_{2}}\left(y_{0}, y_{0}\right)<0
$$

Clearly, by (3.23) and (3.25), we have

$$
\psi^{\prime \prime}\left(r_{0}\right)=\frac{\partial^{2} \psi}{\partial x_{2}^{2}}\left(y_{0}, y_{0}\right)+\frac{\partial^{2} \psi}{\partial y_{2}^{2}}\left(y_{0}, y_{0}\right)+2 \frac{\partial^{2} \psi}{\partial x_{2} \partial y_{2}}\left(y_{0}, y_{0}\right)<0 .
$$

LEMMA 3.4. - Let $G(x, y)$ be the Green function and $y_{1}=r_{1} e_{2}$ for some $a<r_{1}<b$. Then $G\left(r e_{2}, y_{1}\right)$ increases in $r$ for $r \in\left[a, r_{1}\right)$ and decreases in $r$ for $r \in\left(r_{1}, b\right]$.

Proof. - Let

$$
\frac{\partial G}{\partial \theta}\left(x, y_{1}\right)=x_{1} \frac{\partial G}{\partial x_{2}}\left(x, y_{1}\right)-x_{2} \frac{\partial G}{\partial x_{1}}\left(x, y_{1}\right) .
$$

In a neighborhood of $y_{1}, \frac{\partial G}{\partial \theta}\left(x, y_{1}\right)$ satisfies

$$
\frac{\partial G}{\partial \theta}\left(x, y_{1}\right)=\frac{x_{1} r_{1}}{\left|x-y_{1}\right|^{2}}+\text { smooth function }
$$


Since $\frac{\partial G}{\partial \theta}\left(x, y_{0}\right)=0$ for $x \in \Omega^{+} \backslash\left\{y_{1}\right\}$ where $\Omega^{+}=\left\{x \in \Omega \mid x_{1}>0\right\}$, by the maximum principle and (3.26), we have

$$
\frac{\partial G}{\partial \theta}\left(x, y_{1}\right)>0 \text { for } x \in \Omega_{1} .
$$

There,

$$
G\left(x, y_{1}\right)<G\left(|x| e_{2}, y_{1}\right) \quad \text { for } x \in \Omega \text {. }
$$

Now suppose $G\left(r e_{2}, y_{1}\right)$ has a critical point in $\left[a, r_{1}\right)$. Since $G\left(a e_{2}, y_{1}\right)=0$ and $\lim _{r \rightarrow r_{1}} G\left(r e_{2}, y_{1}\right)=+\infty$, we may assume $G\left(r e_{2}, y_{1}\right)$ has a local maximum at $r=r_{0}$. Together with (3.27), we have $G\left(x, y_{1}\right)$ has a local maximum at $x=r_{0} e_{2}$, which violate the strong maximum principle.

Now we are in the position to finish the proof of Theorem 1.4.

Proof of Theorem 1.4. - Suppose that $u_{i}$ blows up at the two points $P$ and $Q$, and $P_{i}$ and $Q_{i}$ are local maximum points of $u_{i}$ near $P$ and $Q$ respectively. As in Theorem 1.2, we first claim that $P_{i}, Q_{i}$ and the origin $O$ is on a straight line. Suppose the claim does not hold. By composing with a rotation, we assume that $P_{i}=\left(t_{i}, s_{i}\right)$ and $Q_{i}=\left(t_{i}, \tilde{s}_{i}\right)$ for some $t_{i}>0$, and $\left|P_{i}-P\right|=\left|Q_{i}-Q\right|$. By following the argument of (2.8), we can show that

$$
w_{0}(x)=u_{i}(x)-u_{i}\left(x^{-}\right)>0 \text { for } x \in \Omega^{+},
$$

where $\Omega^{+}=\left\{x \mid x_{1}>0\right\}$. Then, as in the beginning of this section, we could use the method of rotating planes to yield a contradiction. Hence we conclude that $P_{i}, Q_{i}$ and $O$ are on the same straight line $l_{i}$. The symmetry of $u_{i}$ with respect to $l_{i}$ can be proved similarly.

For simplicity of notations, we may assume both $P_{i}$ and $Q_{i}$ are located on the $x_{2}$-axis. Without loss of generality, we may assume that $P_{i}$ is on the positive $x_{2}$-axis. We claim

$$
Q_{i} \text { is on the negative } x_{2} \text {-axis. }
$$

We prove (3.28) by contradiction. Suppose $Q_{i}$ is on the positive $x_{2}$-axis. Let $P=$ $\left(0, r_{2}\right)$ and $Q=\left(0, r_{1}\right)$. By Theorem 2.1, $\frac{\rho_{i} \mathrm{e}_{i}}{\int_{\Omega} \mathrm{e}^{u_{i}} \mathrm{~d} x}$ converges to $8 \pi(\delta(P)+\delta(Q))$, where $\delta(P)$ is the Dirac measure at $P$. Thus, $u_{i}(x)$ converges to $8 \pi[G(x, P)+G(x, Q)]$ in $C_{\mathrm{loc}}^{2}(\Omega \backslash\{P, Q\})$. By the Pohozaev identity, we have for any unit vector $e$ in $\mathbf{R}^{2}$,

$$
0=\int_{|x-P|=r}\left\langle e, \nabla u_{i}\right\rangle \frac{\partial u_{i}}{\partial v}-\frac{\langle e, v\rangle}{2}\left|\nabla u_{i}\right|^{2} \mathrm{~d} \sigma .
$$

By passing to the limit, we have

$$
0=\int_{|x-P|=r}\langle e, \nabla G\rangle \frac{\partial G}{\partial v}-\frac{\langle e, v\rangle}{2}|\nabla G|^{2} \mathrm{~d} \sigma,
$$

where 


$$
\begin{aligned}
G(x) & =8 \pi[G(x, P)+G(x, Q)] \\
& =4 \log \left(\frac{1}{|x-P|}\right)+8 \pi[\psi(x, P)+G(x, Q)]
\end{aligned}
$$

by (3.19). By a straightforward computation, (3.29) implies

$$
\left\langle e, \nabla_{x}(\psi(x, P)+G(x, Q))\right\rangle=0 \quad \text { at } x=P
$$

as $r \rightarrow 0$. Since $\psi(x, P)$ and $G(x, Q)$ is an even function in $x_{1},(3.30)$ is equivalent to

$$
\frac{\partial G(x, Q)}{\partial x_{2}}+\frac{\partial \psi(x, P)}{\partial x_{2}}=0 \quad \text { at } x=P
$$

that is,

$$
2 \frac{\partial G(P, Q)}{\partial x_{2}}+\frac{\partial \varphi}{\partial x_{2}}(P)=0 .
$$

Similarly, when the Pohozaev identity is applied at $Q$, we have

$$
2 \frac{\partial G}{\partial x_{2}}(Q, P)+\frac{\partial \varphi}{\partial x_{2}}(Q)=0 .
$$

Set $P=\left(0, r_{1}\right)$ and $Q=\left(0, r_{2}\right)$. Suppose

$$
a<r_{2}<r_{1}<b .
$$

Then, by Lemma 3.4, we have

$$
\frac{\partial G}{\partial x_{2}}(Q, P)>0>\frac{\partial G}{\partial x_{2}}(P, Q) .
$$

Thus, by (3.31) and (3.32), we have

$$
\frac{\mathrm{d}}{\mathrm{d} r} \varphi(Q)<0<\frac{\mathrm{d}}{\mathrm{d} r} \varphi(P) .
$$

Let $r_{0}$ be the maximum point $\varphi(r)$. Then by Lemma 3.3, (3.34) implies

$$
r_{1}<r_{0}<r_{2}
$$

which yields a contradiction to (3.33). The other assumption $a<r_{1}<r_{2}<b$ yields a similar contradiction by Lemma 3.3, Lemma 3.4 and (3.32). Hence the claim (3.28) is proved.

Set $\phi_{i}(x)=\frac{\partial u_{i}}{\partial \theta}(x)$. Then $\phi_{i}(x)$ satisfies $\Delta \phi_{i}+\mathrm{e}^{v_{i}} \phi_{i}=0$ in $\Omega^{+}$and $\phi_{i}=0$ on $\partial \Omega^{+}$, where $v_{i}=u_{i}+c_{i}$ for some suitable constant $c_{i}$ and $\Omega^{+}=\left\{\left(x_{1}, x_{2}\right) \in \Omega \mid x_{1}>0\right\}$. Note that $\phi_{i}$ cannot be one sign in $\Omega^{+}$because $u_{i}$ has two local maximum point $P_{i}$ and $Q_{i}$ which are located in the opposite direction of the $x_{2}$-axis. Furthermore, the nodal line $\left\{x \in \Omega^{+} \mid \phi_{i}(x)=0\right\}$ must intersect with the boundary of $\partial \Omega^{+}$at two points at least. This can be seen by the scaling in the following way. 
Set

$$
\tilde{\phi}_{i}(y)=\phi_{i}\left(\mathrm{e}^{-\frac{u_{i}\left(P_{u}\right)}{2}} y\right)
$$

as in (2.17). Then by multiplying a constant, $\tilde{\phi}_{i}(y)$ converges to $c \frac{\partial v}{\partial y_{1}}$ in $C_{\text {loc }}^{2}\left(\mathbf{R}^{2}\right)$, where $c \neq 0$ and $\frac{\partial v}{\partial y_{1}}(y)=-y_{1} /\left(1+\frac{1}{8}|y|^{2}\right)$. Note that $u_{i}$ has a local maximum at $P_{i}$. Thus, $\phi_{i}$ is positive in a neighborhood of $P_{i}$ in $\Omega^{+}$. The same holds for $Q_{i}$ also, that is, $\phi_{i}(x)$ is negative in a neighborhood of $Q_{i}$. This proves that the nodal line of $\phi_{i}$ intersects at least twice with $\partial \Omega^{+}$.

Since $\Omega^{+}$is simply connected, the fact about the nodal line implies that $\Omega^{+}$contains two disjoint, simply-connected subdomain $\Omega_{1}^{+}$and $\Omega_{2}^{+}$where $\phi_{i}(x)$ vanishes on the boundary of $\Omega_{i}^{+}, i=1,2$. By Lemma 3.2,

$$
\rho_{i} \int_{\Omega_{k}^{+}} \mathrm{e}^{u_{i}} \mathrm{~d} x \geqslant 4 \pi
$$

for $k=1,2$. If there exists $\Omega_{k_{0}}^{+}$such that

$$
\rho_{i} \int_{\Omega_{k_{0}}^{+}} \mathrm{e}^{u_{i}} \mathrm{~d} x>4 \pi .
$$

Then $\rho_{i}>16 \pi$ as claim. If (3.35) turns out to be an equality for each $k$, then by using the spherical symmetrization as (3.9), we can prove that $\left|\nabla \phi_{i}\right|(x) \equiv$ constant for $|x|=b$. Since $\phi_{i \theta} \equiv 0$ for $|x|=b$, we have $\phi_{i r}(b, \theta)$ is a constant independent of $\theta$. By integrating $\phi_{i r}(b, \theta)$ along $\theta$, we obtain a contradiction as the last step of the proof of Theorem 1.3. This ends the proof of Theorem 1.4.

\section{Existence of blowing-up solutions}

In this section, we want to prove the existence of blowup solutions of the following equation:

$$
\begin{cases}\Delta u_{i}+\lambda_{i} \mathrm{e}^{u_{i}}=0 & \text { in } \Omega \\ u_{i}=0 & \text { on } \partial \Omega\end{cases}
$$

where $\lambda_{i} \rightarrow 0$. In particular, we want to seek solutions such that

$$
\lambda_{i} \int_{\Omega} \mathrm{e}^{u_{i}} \mathrm{~d} x \rightarrow 8 m \pi
$$

as $\lambda_{i} \rightarrow 0$ when $\Omega$ is a radially symmetric annulus. Write $\lambda_{i}=\rho_{i}\left(\int_{\Omega} \mathrm{e}^{u_{i}} \mathrm{~d} x\right)^{-1}$. Then $u_{i}$ is a solution of (1.4) with $\rho_{i}$ such that $\rho_{i} \rightarrow 8 m \pi$. Our main result in this section is Theorem 4.2 below.

When $\Omega$ is a simply-connected domain under some nondegenerate condition, solutions with one blowup point was constructed in [17,18] and [25]. In general, there 
seems no results about the existence of solutions with one point blowup. For Eq. (1.4) with $\rho \in(8 \pi, 16 \pi)$, solutions were obtained by Ding et al. [12]. However, it is not clear whether their solution would blowup or not as $\rho \downarrow 8 \pi$. (By Theorem 1.4, their solutions remains bounded as $\rho \uparrow 16 \pi$.) On the other hand, since the nonlinear term $\mathrm{e}^{u}>0$ at $u=0$, there always exists a minimal solutions $u_{\lambda} \rightarrow 0$ as $\lambda \rightarrow 0$. It is easy by the wellknown Mountain Pass lemma to prove that for any fixed small $\lambda>0,(4.1)$ possesses a second solution $u_{\lambda}$ at least, and the solution $u_{\lambda}$ must blow up at some point as $\lambda \rightarrow 0$. It is plausible to guess that $u_{\lambda}$ has one single blowup point only. This result might be known to experts. But, there seems no proofs in the literature as far as the authors know. For the sake of the completeness, we present the proof here. When $\Omega$ is an annulus, we also construct solutions with $m$ blowup points which satisfies

$$
\lambda_{i} \int_{\Omega} \mathrm{e}^{u_{i}(x)} \mathrm{d} x>8 m \pi .
$$

In the following, we consider a more general nonlinear term $f(t)$ which satisfies $f(0)>0$ and

$$
\lim _{t \rightarrow+\infty} f(t) \mathrm{e}^{-t}=1 .
$$

Denote $F(t)=\int_{0}^{t} f(s) \mathrm{d} s$. Let $v_{\lambda}$ be the minimal solution of

$$
\begin{cases}\Delta u+\lambda f(u)=0 & \text { in } \Omega, \\ u=0, & \text { on } \partial \Omega .\end{cases}
$$

Set

$$
J_{\lambda}(u)=\frac{1}{2} \int_{\Omega}|\nabla u|^{2}-\lambda \int_{\Omega}\left\{F\left(v_{\lambda}+u\right)-F\left(v_{\lambda}\right)-f\left(v_{\lambda}\right) u\right\}
$$

for $u \in \stackrel{o}{H^{1}}(\Omega)$. Then $J_{\lambda} \in C^{1}\left(\stackrel{o}{H^{1}}(\Omega)\right)$ and satisfies the Palais-Smale condition. Suppose that there is $e_{\lambda} \in \stackrel{o}{H^{1}}(\Omega)$ such that $J_{\lambda}\left(e_{\lambda}\right) \leqslant 0$. Let

$$
c_{\lambda} \equiv \inf _{\gamma} \max _{0 \leqslant t \leqslant 1} J_{\lambda}(\gamma(t))
$$

where $\gamma$ is any path connecting 0 and $e_{\lambda}$. The well-known Mountain Pass Lemma says that $c_{\lambda}$ is a critical value of $J_{\lambda}$. Let $w_{\lambda}$ be a critical point of $J_{\lambda}$ with $J_{\lambda}\left(w_{\lambda}\right)=c_{\lambda}$. Then $u_{\lambda} \equiv v_{\lambda}+w_{\lambda}$ is a solution of (4.1). It is easy to see that solutions obtained in this manner must blow up as $\lambda \rightarrow 0$. We will prove that, by choosing a particular $e_{\lambda}, u_{\lambda}$ blows up at a single point as $\lambda \rightarrow 0$.

THEOREM 4.1. - Let $u_{\lambda}$ be the solution described above. Then $u_{\lambda}$ blows up at a single point as $\lambda \rightarrow 0$. 
Proof. - Let $B$ be a ball contained in $\Omega$, and $U_{\lambda}^{\varepsilon}$ be the large-norm solution of

$$
\begin{cases}\Delta u+\lambda \mathrm{e}^{(1-\varepsilon) u}=0, & \\ u(x)>0 & \text { in } B, \\ u(x)=0 & \text { on } \partial B,\end{cases}
$$

where $\varepsilon$ is a fixed small positive number. Then we have

$$
\begin{gathered}
\lambda \int_{B} \mathrm{e}^{(1-\varepsilon) U_{\lambda}^{\varepsilon}}=\frac{8 \pi}{1-\varepsilon}(1+\mathrm{o}(1)) \quad \text { as } \lambda \downarrow 0, \\
\lambda \int_{B} U_{\lambda}^{\varepsilon} \mathrm{e}^{(1-\varepsilon) U_{\lambda}^{\varepsilon}}=\int_{B}\left|\nabla U_{\lambda}^{\varepsilon}\right|^{2}=\frac{16 \pi}{(1-\varepsilon)^{2}}(1+\mathrm{o}(1)) \log \frac{1}{\lambda},
\end{gathered}
$$

and

$$
\int_{B} U_{\lambda}^{\varepsilon} \mathrm{d} x \leqslant c
$$

where $c$ is a constant independent of $\lambda$ and $\varepsilon$.

Extend $U_{\lambda}^{\varepsilon}$ to be zero outside $B$, then $U_{\lambda}^{\varepsilon} \in \stackrel{o}{H^{1}}(\Omega)$. We have

$$
\begin{aligned}
J_{\lambda}\left((1+\varepsilon) U_{\lambda}^{\varepsilon}\right)= & \frac{(1+\varepsilon)^{2}}{2} \int_{\omega}\left|\nabla U_{\lambda}^{\varepsilon}\right|^{2} \mathrm{~d} x-\lambda \int_{\Omega}\left[F\left((1+\varepsilon) U_{\lambda}^{\varepsilon}+v_{\lambda}\right)\right. \\
& \left.-F\left(v_{\lambda}\right)-f\left(v_{\lambda}\right)(1+\varepsilon) U_{\lambda}^{\varepsilon}\right] \mathrm{d} x .
\end{aligned}
$$

When the value of $U_{\lambda}^{\varepsilon}(x)$ is large, by (4.3),

$$
\begin{aligned}
F\left((1+\varepsilon) U_{\lambda}^{\varepsilon}+v_{\lambda}\right) & \geqslant \mathrm{e}^{(1-\varepsilon)\left[(1+\varepsilon) U_{\lambda}^{\varepsilon}+v_{\lambda}\right]}-c_{1}(\varepsilon) \\
& \geqslant c_{2} \varepsilon^{2}\left(U_{\lambda}^{\varepsilon}\right)^{2} \mathrm{e}^{(1-\varepsilon) U_{\lambda}^{\varepsilon}}-c_{1}(\varepsilon) .
\end{aligned}
$$

When $\varepsilon$ is fixed, we have

$$
\int_{\Omega}\left(U_{\lambda}^{\varepsilon}\right)^{2} \mathrm{e}^{(1-\varepsilon) U_{\lambda}^{\varepsilon}} / \int_{\Omega} U_{\lambda}^{\varepsilon} \mathrm{e}^{(1-\varepsilon) U_{\lambda}^{\varepsilon}} \rightarrow+\infty
$$

as $\lambda \rightarrow 0$. By observing that $v_{\lambda}$ is uniformly bounded in $\lambda$, it is easy to see that

$$
J_{\lambda}\left((1+\varepsilon) U_{\lambda}^{\varepsilon}\right) \rightarrow-\infty \quad \text { as } \lambda \downarrow 0 .
$$

Let $e_{\lambda}=(1+\varepsilon) U_{\lambda}^{\varepsilon}$, then for small $\lambda$,

$$
J_{\lambda}\left(e_{\lambda}\right) \leqslant 0,
$$

and let

$$
c_{\lambda} \equiv \inf _{\gamma} \max _{0 \leqslant t \leqslant 1} J_{\lambda}(\gamma(t))
$$


where $\gamma$ is any continuous path connecting 0 and $e_{\lambda}$. By the Mountain Pass Lemma, we know that $c_{\lambda}$ is a critical value. Let $w_{\lambda}$ be one of the critical points of $J_{\lambda}$ with $J_{\lambda}\left(w_{\lambda}\right)=c_{\lambda}$. Then $u_{\lambda}=w_{\lambda}+v_{\lambda}$ is a solution of (4.4).

To compute $\int_{\Omega}\left|\nabla u_{\lambda}\right|^{2}$, one have

$$
\lambda \int_{\Omega} u_{\lambda} f\left(u_{\lambda}\right) \mathrm{d} x=\int_{\Omega}\left|\nabla u_{\lambda}\right|^{2}=2 J_{\lambda}\left(w_{\lambda}\right)+2 \lambda \int_{\Omega} F\left(u_{\lambda}\right)-F\left(v_{\lambda}\right)-f\left(v_{\lambda}\right) w_{\lambda}+\mathrm{O}(1) .
$$

Fixed $\varepsilon>0$, one have

$$
\lambda \int_{\Omega} F\left(u_{\lambda}\right)<\varepsilon \lambda \int_{\Omega} u_{\lambda} F\left(u_{\lambda}\right)<c \varepsilon \lambda \int_{\Omega} u_{\lambda} f\left(u_{\lambda}\right)
$$

for all small $\lambda$. Hence

$$
\begin{aligned}
\mathrm{O}(1)+\int_{\Omega}\left|\nabla u_{\lambda}\right|^{2} & \leqslant \frac{2}{1-c \varepsilon} J_{\lambda}\left(w_{\lambda}\right) \\
& \leqslant \frac{2}{1-c \varepsilon} \max _{0 \leqslant t \leqslant 1+\varepsilon} J_{\lambda}\left(t U_{\lambda}^{\varepsilon}\right) \\
& \leqslant \frac{(1+\varepsilon)^{2}}{1-c \varepsilon} \int_{\frac{\Omega}{\Omega}}\left|\nabla U_{\lambda}^{\varepsilon}(x)\right|^{2} \mathrm{~d} x+\mathrm{O}(1) \\
& \leqslant \frac{(1+\varepsilon)^{2}}{(1-\varepsilon)^{2}(1-c \varepsilon)} 16 \pi \log 1 / \lambda(1+\mathrm{o}(1)),
\end{aligned}
$$

by (4.5). Let $\varepsilon$ satisfy

$$
\frac{(1+\varepsilon)^{2}}{(1-\varepsilon)^{2}(1-c \varepsilon)}<\frac{3}{2}
$$

By Lemma 4.3 (below), we know that the blow-up point of $u_{\lambda}$ consist of one single-point only and $\lambda \int_{\Omega} f(u) \mathrm{d} x=8 \pi(1+\mathrm{o}(1))$. Hence the proof of Theorem 4.1 is complete.

THEOREM 4.2. - Let $\Omega$ be a radially symmetric annulus and $f(t)=\mathrm{e}^{t}$. Let $m$ be any positive integer. Then for small $\lambda>0$, there exists a solutions $u_{\lambda}$ of (4.1) such that

$$
\lambda \int_{\Omega} \mathrm{e}^{u_{\lambda}} \mathrm{d} x>8 m \pi, \quad \text { and } \lim _{\lambda \rightarrow 0} \lambda \int_{\Omega} \mathrm{e}^{u_{\lambda}} \mathrm{d} x=8 m \pi .
$$

Proof. - We will prove Theorem 4.2 in a similar manner. Let $T_{m}$ be the rotation with an angle $2 \pi / m$. Consider $J_{\lambda}$ of (4.5) in the pace $\stackrel{\circ}{H}^{1}{ }_{m}(\Omega)$, where $\stackrel{\circ}{H^{1}}{ }_{m}(\Omega)=\{u \in$ $\left.\stackrel{\circ}{H^{1}}(\Omega) \mid u\left(T_{m}(x)\right)=u(x)\right\}$. As in the proof of Theorem 4.1, we construct a $U_{\lambda}^{\varepsilon} \in \stackrel{\circ}{H}^{1}{ }_{m}(\Omega)$ such that

$$
\lambda \int_{\Omega} U_{\lambda}^{\varepsilon} \mathrm{e}^{(1-\varepsilon) U_{\lambda}^{\varepsilon}}=\frac{16 m \pi}{(1-\mathrm{e})^{2}} \log \frac{1}{\lambda}(1+\mathrm{o}(1)) .
$$


And, we have

$$
J_{\lambda}\left((1+\varepsilon) U_{\lambda}^{\varepsilon}\right) \rightarrow-\infty \quad \text { as } \lambda \rightarrow 0 .
$$

Let $e_{\lambda}=(1+\varepsilon) U_{\lambda}^{\varepsilon}$, and

$$
c_{\lambda}=\inf _{\gamma} \max _{0 \leqslant t \leqslant 1} J_{\lambda}(\gamma(t))
$$

where $\gamma$ is any continuous path in $\stackrel{\circ}{H}^{1}{ }_{m}(\Omega)$ connecting 0 and $e_{\lambda}$. Hence there exists a critical point $w_{\lambda} \in \stackrel{\circ}{H}^{1}{ }_{m}(\Omega)$ with $J_{\lambda}\left(w_{\lambda}\right)=c_{\lambda}$. Then $u_{\lambda}=v_{\lambda}+w_{\lambda}$ is a solution of (4.4), and invariant under $T_{m}$. In a similar manner as shown above, we can choose $\varepsilon$ small so that

$$
\int_{\Omega}\left|\nabla u_{\lambda}\right|^{2} \leqslant 24 m \pi \log 1 / \lambda(1+o(1)) \quad \text { as } \lambda \downarrow 0 .
$$

Since the number of blow-up points of $u_{\lambda}$ is a multiple of $m$, by (4.15) of Lemma 4.3 (below), the above inequality implies that the blow-up set of $u_{\lambda}$ consists of $m$ points. Hence, $\lambda \int_{\Omega} f\left(u_{\lambda}\right) \mathrm{d} x=8 \pi m(1+\mathrm{o}(1))$ as $\lambda \downarrow 0$.

We give a sketch of the proof for (4.11). For simplicity, we assume $m=3$. The proof for the general case is similar. Now suppose $u_{i}$ has the local maximum at $P_{i}, Q_{i}, \tilde{Q}_{i}$, where $P_{i}$ is on the positive $x_{1}$-axis and $Q_{i}, \tilde{Q}_{i}$ are located at the rays having the angle $2 \pi / 3$ and $4 \pi / 3$ to the positive $x_{1}$-axis respectively. we want to prove $u_{i}(x)$ is symmetric with respect to the $x_{1}$-axis. Note that $Q_{i}$ is the reflection point of $\tilde{Q}_{i}$ with respect to the $x_{1}$-axis.

Set $w\left(x_{1}, x_{2}\right)=u_{i}\left(x_{1}, x_{2}\right)-u_{i}\left(x_{1},-x_{2}\right)$ for $x_{2} \geqslant 0$. Then $w$ satisfies

$$
\left\{\begin{array}{l}
\Delta w+b(x) w(x)=0 \quad \text { in } \Omega^{+}=\left\{x \in \Omega \mid x_{2} \geqslant 0\right\}, \\
\left.w\right|_{\partial \Omega^{+}}=0 .
\end{array}\right.
$$

Suppose $w(x) \not \equiv 0$ in $\Omega^{+}$. Then it can be proved as (2.15) such that for any $\delta>0$,

$$
\sup _{\Omega^{+}(\delta)}|w(x)|=\mathrm{o}(1) N_{i}
$$

holds, where o(1) $\rightarrow 0$ as $i \rightarrow+\infty$,

$$
N_{i}=\max _{\Omega^{+}}|w(x)|
$$

and

$$
\Omega^{+}(\delta)=\left\{x \in \Omega^{+}|| x-Q_{i} \mid \geqslant \delta \text { and }\left|x-P_{i}\right| \geqslant \delta\right\} .
$$

Now suppose the maximum of $w$ occures near $Q_{i}$. Note that $w(x)=0$ for $x \in$ $\left\{\left(t \cos \frac{2 \pi}{3}, t \sin \frac{2 \pi}{3}\right), t \geqslant 0\right\}$ because $u_{i}$ is invariant under the group action of $T_{3}$. By rotation and translation, we assume $Q_{i}=(0,0), w(x)$ satisfies

$$
\left\{\begin{array}{l}
\Delta w(x)+b(x) w(x)=0 \quad \text { for }|x| \leqslant \delta \text { and } x_{2} \geqslant 0, \\
w\left(x_{1}, 0\right)=0
\end{array}\right.
$$


and the maximum of $w(x)$ occures near $Q_{i}$, say,

$$
\left|w\left(x_{i}\right)\right|=N_{i} .
$$

By scaling as (2.17), we consider $\tilde{w}(y)=w\left(\mathrm{e}^{-u_{i}\left(Q_{i}\right) / 2} y\right)$ for $|y| \leqslant \mathrm{e}^{u_{i}\left(Q_{i}\right) / 2} \delta$. Then $\tilde{w}(y)$ satisfies

$$
\Delta \tilde{w}+\tilde{b}(y) \tilde{w}(y)=0 \quad \text { for }|y| \leqslant \mathrm{e}^{\frac{u_{i}\left(Q_{i}\right)}{2} \delta}
$$

with

$$
|\tilde{b}(y)| \leqslant c\left(1+|y|^{2}\right)^{-2} .
$$

We apply the Green representation formula (2.19) to prove $\mathrm{e}^{u_{i}\left(Q_{i}\right) / 2}\left|x_{i}\right|$ is bounded. Furthermore, $N_{i}^{-1} \tilde{w}(x)$ tends to some function $\tilde{w}(x)$ in $C_{\text {loc }}^{2}\left(\mathbf{R}^{2}\right)$, where $\tilde{w}$ satisfies

$$
\left\{\begin{array}{l}
\Delta \tilde{w}+\mathrm{e}^{v(x)} \tilde{w}=0 \quad \text { in } \mathbf{R}_{+}^{2} \\
\tilde{w}\left(x_{1}, 0\right)=0
\end{array}\right.
$$

By the reflection, we have

$$
\tilde{w}(x)=\frac{c x_{1}}{1+\frac{1}{8}|x|^{2}} \quad \text { in } \mathbf{R}_{+}^{2}
$$

for some constant $c \neq 0$. On the other hand,

$$
\tilde{w}(0)=|\nabla \tilde{w}(0)|=0
$$

because $u_{i}$ has local maximum at $Q_{i}$ and $\tilde{Q}_{i}$. Then

$$
0=\frac{\partial \tilde{w}}{\partial x_{1}}(0,0)=c \neq 0
$$

yields a contradiction. Hence the maximum of $w$ must occur near $P_{i}$. By using the same argument, it also yields a contradiction. Therefore the symmetry of $u_{i}$ is proved.

Let $\varphi=\partial u_{i} / \partial \theta$. Then $\varphi$ vanishes at the rays $\{(t \cos \theta, t \sin \theta) \mid t \geqslant 0\}$ with $\theta=$ $k \pi / 3, k=0,1, \ldots, 5$. By the isoperimetric inequality (see the proof of Theorem 1.3), we have

$$
\lambda_{i} \int_{0 \leqslant \theta \leqslant \frac{\pi}{3}} \mathrm{e}^{u_{i}(x)} \mathrm{d} x>4 \pi .
$$

Therefore

$$
\lambda_{i} \int_{\Omega} \mathrm{e}^{u_{i}}(x) \mathrm{d} x>24 \pi
$$

as claimed.

LEMMA 4.3. - Suppose that $u_{i}$ is a sequence of solutions of (4.4) with $\lambda_{i} \rightarrow 0$. Then after passing to a subsequence (still denoted by $u_{i}$ ), one of the following statements hold. 
(i) $\lambda_{i} \int_{\Omega} f\left(u_{i}\right) \mathrm{d} x \rightarrow+\infty$ as $i \rightarrow+\infty$. In this case, $u_{i}(x) \rightarrow+\infty$ uniformly in any compact set of $\Omega$,

(ii) $\lim _{i \rightarrow+\infty} \lambda_{i} \int_{\Omega} f\left(u_{i}\right) \mathrm{d} x=8 m \pi$ for some integer $m \in\{0,1,2, \ldots\}$. Furthermore, we have (a) if $m=0$, then $u_{i}(x) \rightarrow 0$ uniformly for $x \in \bar{\Omega}$, (b) if $m>0$, then $u_{i}$ has exactly $m$ blowup points $\left\{P_{1}, \ldots, P_{m}\right\}$ and $\lambda_{i} f\left(u_{i}(x)\right) \rightarrow 8 \pi \sum_{j=1}^{m} \delta_{P_{j}}$ with $\delta_{p_{j}}$ being the Dirac measure at $P_{j}$. Moreover, as $i \rightarrow \infty$

$$
\lambda_{i} \int_{\left|P_{j}-x\right| \leqslant \delta} f\left(u_{i}(x)\right) u_{i}(x) \mathrm{d} x=16 m \pi \log \frac{1}{\lambda_{i}}(1+\mathrm{o}(1)) .
$$

Proof. - The claims of (i) and (ii) are well-known now except (4.15). (4.15) is a consequence of the estimate of local maximum of $u_{i}$ near each blowup point $P_{j}$ :

$$
\max _{\left|x-P_{j}\right| \leqslant \delta} u_{i}(x)=2 \log \frac{1}{\lambda_{i}}(1+\mathrm{o}(1))
$$

for each $j$ and small $\delta>0$. The upper bound of $\max _{\left|x-P_{j}\right| \leqslant \delta} u_{i}(x)$ can be obtained by the so-called "sup + inf" inequality which was proved in [3] and [8]. The lower bound can be obtained by the Green representation formulas and the scaling method. For the details of proof, we refer to [9].

\section{REFERENCES}

[1] Bandle C., Isoperimetric Inequalities and Applications, Pitman, Boston, 1980.

[2] Brezis H., Merle F., Uniform estimates and blow-up behavior for solutions of $-\Delta u=$ $V(x) \mathrm{e}^{u}$ in two dimensions, Comm. Partial Differential Equations 16 (1991) 1223-1254.

[3] Brezis H., Li Y.Y., Shafrir I., A sup + inf inequality for some nonlinear elliptic equations involving exponential nonlinearities, J. Functional Anal. 115 (1993) 344-358.

[4] Caffarelli L., Yang Y., Vortex condensation in the Chern-Simons Higgs model: An existence theorem, Comm. Math. Phys. 168 (1995) 321-336.

[5] Chanillo S., Kiessling M., Rotational symmetry of solutions of some nonlinear problems in statistical mechanics and in geometry, Comm. Math. Phys. 160 (1994) 217-238.

[6] Caglioti E., Lions P.L., Marchioro C., Pulvirenti M., A special class of stationary flows for two-dimensional Euler equations: A statistical mechanics description, Comm. Math. Phys. 143 (1992) 501-525.

[7] Caglioti E., Lions P.L., Marchioro C., Pulvirenti M., A special class of stationary flows for two-dimensional Euler equations: A statistical mechanics description, part II, Comm. Math. Phys. 174 (1995) 229-260.

[8] Chen C.C., Lin C.S., A sharp sup + inf inequality for a nonlinear equation in $\mathbf{R}^{2}$, Comm. Anal. Geom. 6 (1998) 1-19.

[9] Chen C.C., Lin C.S., Singular limits of a nonlinear eigenvalue problem in two dimensions, preprint.

[10] Chen W., Li C., Classification of solutions of some nonlinear elliptic equations, Duke Math. J. 63 (1991) 615-623.

[11] Ding W., Jost J., Li J., Wang G., The differential equation $\Delta u=8 \pi-8 \pi h \mathrm{e}^{u}$ on a compact Riemann surface, Asian J. Math. 1 (1997) 230-248.

[12] Ding W., Jost J., Li J., Wang G., Existence results for mean field equations, preprint. 
[13] Gidas B., Ni W.M., Nirenberg L., Symmetry of positive solutions of nonlinear elliptic equations in $\mathbf{R}^{n}$, in: Nachbin L. (Ed.), Math. Anal. and Applications, Part A, Advances in Math. Suppl. Studies 7A, Academic Press, New York, 1981, pp. 369-402.

[14] Li Y.Y., Harnack type inequality: the method of moving planes, Comm. Math. Phys. 200 (1999) 421-444.

[15] Li Y.Y., Shafrir I., Blowup analysis for solutions $-\Delta u=V \mathrm{e}^{u}$ in dimension two, Indiana Univ. Math. J. 43 (1994) 1255-1270.

[16] Lin C.S., The topological degree for the mean field equation on $S^{2}$, Duke Math. J. 104 (2000) 501-536.

[17] Moseley J.L., Asymptotic solutions for a Dirichlet problem with an exponential nonlinearity, SIAM J. Math. Anal. 14 (1983) 719-735.

[18] Moseley J.L., A two-dimensional Dirichlet problem with an exponential nonlinearity, SIAM J. Math. Anal. 14 (1983) 934-946.

[19] Nagasaki K., Suzuki T., Asymptotic analysis for two-dimensional elliptic eigenvalue problems with exponentially dominated nonlinearity, Asymptotic Analysis 3 (1990) 173188.

[20] Nolasco M., Tarantello G., On a sharp type inequality on two dimensional compact manifolds, Arch. Rational Mech. Anal. 145 (1998) 161-195.

[21] Spruck J., Yang Y., Topological solutions in the self-dual Chern-Simons theory: existence and approximation, Ann. Inst. H. Poincáre Anal. Non Linéaire 12 (1995) 75-97.

[22] Struwe M., Tarantello G., On multivortex solutions in Chern-Simons Gauge theory, Boll. Unione Math. Ital. Sez. B Artic. Ric. Mat. 8 (1) (1998) 109-121.

[23] Suzuki T., Global analysis for a two-dimensional elliptic eigenvalues problem with the exponential nonlinearity, Ann. Inst. Henri Poincare, Anal. Non-Linéaire 9 (1992) 367398.

[24] Tarantello G., Multiple condensate solutions for the Chern-Simons-Higgs theory, J. Math. Phys. 37 (1996) 3769-3796.

[25] Weston V.H., On the asymptotic solution of a partial differential equation with an exponential nonlinearity, SIAM J. Math. Anal. 9 (1978) 1030-1053. 